Internat. J. Math. \& Math. Sci.

Vol. 24, No. 5 (2000) 351-359

S0161171200004075

(C) Hindawi Publishing Corp.

\title{
QUADRATIC FUNCTIONAL EQUATIONS OF PEXIDER TYPE
}

\author{
SOON-MO JUNG
}

(Received 15 October 1999)

\begin{abstract}
First, the quadratic functional equation of Pexider type will be solved. By applying this result, we will also solve some functional equations of Pexider type which are closely associated with the quadratic equation.
\end{abstract}

Keywords and phrases. Quadratic functional equation, quadratic equation of Pexider type.

2000 Mathematics Subject Classification. Primary 39B52.

1. Introduction. It is easy to see that the quadratic function $f(x)=x^{2}$ is a solution of each of the following functional equations

$$
\begin{aligned}
& f(x+y)+f(x-y)=2 f(x)+2 f(y), \\
& f(x+y+z)+f(x)+f(y)+f(z)=f(x+y)+f(y+z)+f(z+x), \\
& f(x-y-z)+f(x)+f(y)+f(z)=f(x-y)+f(y+z)+f(z-x), \\
& f(x+y+z)+f(x-y+z)+f(x+y-z)+f(-x+y+z) \\
& =4 f(x)+4 f(y)+4 f(z) .
\end{aligned}
$$

So, it is natural that each equation is called a quadratic functional equation. In particular, every solution of the "original" quadratic functional equation (1.1) is said to be a quadratic function.

It is well known that a function $f$ between real vector spaces is quadratic if and only if there exists a unique symmetric biadditive function $B$ such that $f(x)=B(x, x)$ for all $x$ (see $[1,2,3,6,7]$ ).

The functional equation (1.2) was first solved by Kannappan. In fact, he proved that a functional on a real vector space is a solution of (1.2) if and only if there exist a symmetric biadditive function $B$ and an additive function $A$ such that $f(x)=B(x, x)+$ $A(x)$ for any $x$ (see [6]), while the author investigated, in [4,5], the stability problems of (1.2) and (1.3) on restricted domains and applied the result to the study of asymptotic behaviors of the quadratic functions. Moreover, the quadratic functional equation (1.2) was "pexiderized" and solved by Kannappan (see [6]).

The functional equation (1.2) is different from (1.3), and (1.4) in a sense that every non-zero additive function is a solution of (1.2), but it is not a solution of either (1.3) or (1.4).

In Section 2, we will show that each of the functional equations (1.1), (1.3), and (1.4) is equivalent to the other. The general solutions of the quadratic functional equation of Pexider type, 


$$
f_{1}(x+y)+f_{2}(x-y)=2 f_{3}(x)+2 f_{4}(y)
$$

will be investigated in Section 3. In Sections 4 and 5, the result of Section 3 will be applied to the study of the general solutions of the functional equations

$$
\begin{array}{r}
f_{1}(x-y-z)+f_{2}(x)+f_{3}(y)+f_{4}(z)=f_{5}(x-y)+f_{6}(y+z)+f_{7}(z-x) \\
f_{1}(x+y+z)+f_{2}(x-y+z)+f_{3}(x+y-z)+f_{4}(-x+y+z) \\
=4 f_{5}(x)+4 f_{6}(y)+4 f_{7}(z)
\end{array}
$$

which are "pexiderized" forms of (1.3) and (1.4).

2. Solutions of equations (1.3) and (1.4). It is a natural thing to expect that both the functional equations (1.3) and (1.4) are equivalent to the "original" quadratic equation (1.1). In fact, it is so as we shall see in the following theorem.

THEOREM 2.1. Let $X$ and $Y$ be vector spaces over fields of characteristic different from 2, respectively. If $f: X \rightarrow Y$ satisfies the functional equations (1.1), (1.3), and (1.4), then each of the equations (1.1), (1.3), and (1.4) is equivalent to the other.

Proof. First, we will prove the equivalence of (1.1) and (1.3). Suppose (1.3) holds. If we put $x=y=z=0$ in (1.3), we get $f(0)=0$. By putting $y=z=0$ in (1.3) we see that every solution of (1.3) is even.

Replacing $z$ by $-y$ in (1.3) and using the evenness of $f$ and $f(0)=0$, we can transform (1.3) into (1.1).

Conversely, assume a function $f: X \rightarrow Y$ is a solution of (1.1). Clearly, we see that $f(0)=0, f$ is even,

$$
f(y)+f(z)=2 f\left(\frac{y+z}{2}\right)+2 f\left(\frac{y-z}{2}\right), \quad f\left(\frac{x}{2}\right)=\frac{1}{4} f(x) .
$$

So,

$$
\begin{aligned}
f(x-y-z)+f(x)+f(y)+f(z) & =2 f\left(x-\frac{y+z}{2}\right)+2 f\left(\frac{y-z}{2}\right)+4 f\left(\frac{y+z}{2}\right) \\
& =f(x-y)+f(x-z)+f(y+z) .
\end{aligned}
$$

This implies the equivalence of the functional equations (1.1) and (1.3).

It remains to prove the equivalence of (1.1) and (1.4). If we put $x=y=z=0$ in (1.4), we get $f(0)=0$. By putting $y=z=0$ in (1.4), we see that every solution of (1.4) is even. By putting $z=0$ in (1.4) and using the evenness of $f$ and $f(0)=0$, we can transform (1.4) into (1.1).

Now, suppose a function $f: X \rightarrow Y$ satisfies (1.1) for all $x, y, z \in X$. Then $f$ is even. Hence, we get

$$
\begin{aligned}
& f(x+y+z)+f(x-y+z)+f(x+y-z)+f(-x+y+z) \\
& \quad=2 f(x+z)+2 f(y)+2 f(x-z)+2 f(y)=2(2 f(x)+2 f(z))+4 f(y) .
\end{aligned}
$$

This means the equivalence of (1.1) and (1.4). 
3. Solutions of equation (1.5). In the following theorem, we will find out the general solutions of the functional equation (1.5) which is a "pexiderized" form of the quadratic functional equation (1.1). This result will be applied to the proofs of Theorems 4.1 and 5.1 in which the quadratic functional equations of Pexider type, (1.6) and (1.7), are solved.

THEOREM 3.1. Let $X$ and $Y$ be vector spaces over fields of characteristic different from 2 , respectively. The functions $f_{1}, f_{2}, f_{3}, f_{4}: X \rightarrow Y$ satisfy the functional equation (1.5) for all $x, y \in X$ if and only if there exist a quadratic function $Q: X \rightarrow Y$, additive functions $a_{1}, a_{2}: X \rightarrow Y$, and constants $c_{1}, c_{2}, c_{3}, c_{4} \in Y$ such that

$$
\begin{aligned}
& f_{1}(x)=Q(x)+a_{1}(x)+a_{2}(x)+c_{1}, \\
& f_{2}(x)=Q(x)+a_{1}(x)-a_{2}(x)+c_{2}, \\
& f_{3}(x)=Q(x)+a_{1}(x)+c_{3}, \\
& f_{4}(x)=Q(x)+a_{2}(x)+c_{4}
\end{aligned}
$$

with

$$
c_{1}+c_{2}=2 c_{3}+2 c_{4} .
$$

Proof. We first assume that $f_{1}, f_{2}, f_{3}, f_{4}$ are solutions of the functional equation (1.5). If we define $c_{i}=f_{i}(0)$, then we can verify by putting $x=y=0$ in (1.5) that the relation (3.2) is true.

We now define $F_{i}(x)=f_{i}(x)-c_{i}$ and denote by $F_{i}^{e}$ and $F_{i}^{o}$ the even part and the odd part of $F_{i}(i=1,2,3,4)$.

Clearly, $F_{1}, F_{2}, F_{3}, F_{4}$ are solutions of (1.5). By replacing $x$ and $y$ by $-x$ and $-y$ in (1.5) for the $F_{i}$ 's and then adding (subtracting) the resulting equation to (from) the original equation (1.5), we have

$$
\begin{aligned}
& F_{1}^{e}(x+y)+F_{2}^{e}(x-y)=2 F_{3}^{e}(x)+2 F_{4}^{e}(y), \\
& F_{1}^{o}(x+y)+F_{2}^{o}(x-y)=2 F_{3}^{o}(x)+2 F_{4}^{o}(y) .
\end{aligned}
$$

By putting $y=0, x=0, y=x$, and $y=-x$ in (3.3) separately, we get

$$
\begin{aligned}
F_{1}^{e}(x)+F_{2}^{e}(x)=2 F_{3}^{e}(x), & F_{1}^{o}(x)+F_{2}^{o}(x)=2 F_{3}^{o}(x), \\
F_{1}^{e}(x)+F_{2}^{e}(x)=2 F_{4}^{e}(x), & F_{1}^{o}(x)-F_{2}^{o}(x)=2 F_{4}^{o}(x), \\
F_{1}^{e}(2 x)=2 F_{3}^{e}(x)+2 F_{4}^{e}(x), & F_{1}^{o}(2 x)=2 F_{3}^{o}(x)+2 F_{4}^{o}(x), \\
F_{2}^{e}(2 x)=2 F_{3}^{e}(x)+2 F_{4}^{e}(x), & F_{2}^{o}(2 x)=2 F_{3}^{o}(x)-2 F_{4}^{o}(x) .
\end{aligned}
$$

From (3.4) and (3.5) we obtain $F_{3}^{e}=F_{4}^{e}$. Similarly, by (3.6) and (3.7) we may conclude that $F_{1}^{e}=F_{2}^{e}$. Applying these facts to (3.3) and putting $y=0$ in the resulting equation, we see that there exists a quadratic function $Q: X \rightarrow Y$ with

$$
F_{1}^{e}=F_{2}^{e}=F_{3}^{e}=F_{4}^{e}=Q .
$$

By the second equations in (3.4) and (3.5) we have

$$
F_{1}^{o}=F_{3}^{o}+F_{4}^{o}, \quad F_{2}^{o}=F_{3}^{o}-F_{4}^{o} .
$$


From the second equations in (3.6) and (3.7) and from (3.9) it follows that

$$
\begin{aligned}
& F_{3}^{o}(2 x)+F_{4}^{o}(2 x)=2 F_{3}^{o}(x)+2 F_{4}^{o}(x), \\
& F_{3}^{o}(2 x)-F_{4}^{o}(2 x)=2 F_{3}^{o}(x)-2 F_{4}^{o}(x) .
\end{aligned}
$$

By the last two equations in (3.10) we get

$$
F_{3}^{o}(2 x)=2 F_{3}^{o}(x), \quad F_{4}^{o}(2 x)=2 F_{4}^{o}(x) .
$$

By using (3.9) and the second equation in (3.3), we obtain

$$
F_{3}^{o}(x+y)+F_{4}^{o}(x+y)+F_{3}^{o}(x-y)-F_{4}^{o}(x-y)=2 F_{3}^{o}(x)+2 F_{4}^{o}(y) .
$$

If we replace $y$ in (3.12) by $-y$ and if we add the resulting equation to (3.12), then by (3.11) we get

$$
F_{3}^{o}(x+y)+F_{3}^{o}(x-y)=F_{3}^{o}(2 x),
$$

that is, $F_{3}^{o}$ is an additive function, say

$$
F_{3}^{o}=a_{1},
$$

where $a_{1}: X \rightarrow Y$ is an additive function. By (3.11) and (3.12) we may conclude that $F_{4}^{o}$ is also additive, say

$$
F_{4}^{o}=a_{2},
$$

where $a_{2}: X \rightarrow Y$ is an additive function.

Consequently, the relations in (3.1) are true in view of the equations in (3.8), (3.9), (3.14), and (3.15).

Conversely, if there exist a quadratic function $Q: X \rightarrow Y$, additive functions $a_{1}, a_{2}$ : $X \rightarrow Y$, and constants $c_{1}, c_{2}, c_{3}, c_{4} \in Y$ with the relations in (3.1) and (3.2), we may easily check that the $f_{i}$ 's satisfy (1.5).

4. Solutions of equation (1.6). In this section, we will solve the functional equation (1.6) which is a "pexiderized" form of (1.3).

THEOREM 4.1. Let $X$ and $Y$ be vector spaces over fields of characteristic different from 2 , respectively. The functions $f_{i}: X \rightarrow Y(i=1, \ldots, 7)$ satisfy the functional equation (1.6) if and only if there exist a quadratic function $Q: X \rightarrow Y$, constants $c_{i} \in Y$ $(i=1, \ldots, 7)$, and additive functions $a_{i}: X \rightarrow Y(i=1, \ldots, 4)$ such that

$$
\begin{aligned}
& f_{1}(x)=Q(x)+a_{1}(x)-a_{2}(x)-a_{3}(x)+c_{1}, \\
& f_{2}(x)=Q(x)-a_{1}(x)+2 a_{3}(x)+a_{4}(x)+c_{2}, \\
& f_{3}(x)=Q(x)+a_{1}(x)-a_{4}(x)+c_{3}, \\
& f_{4}(x)=Q(x)+a_{1}(x)+a_{2}(x)-a_{3}(x)+c_{4}, \\
& f_{5}(x)=Q(x)+a_{4}(x)+c_{5}, \\
& f_{6}(x)=Q(x)+a_{2}(x)+a_{3}(x)+c_{6}, \\
& f_{7}(x)=Q(x)+a_{2}(x)-a_{3}(x)+c_{7}
\end{aligned}
$$


with

$$
c_{1}+c_{2}+c_{3}+c_{4}=c_{5}+c_{6}+c_{7} .
$$

Proof. First, assume that the $f_{i}$ 's are solutions of (1.6). Define $c_{i}=f_{i}(0)$ for $i=$ $1, \ldots, 7$. Putting $x=y=z=0$ in (1.6) yields relation (4.2). For $i=1, \ldots, 7$ define $F_{i}(x)=$ $f_{i}(x)-c_{i}$ for all $x \in X$. Then we have $F_{i}(0)=0$ for $i=1, \ldots, 7$. It follows from (1.6) and (4.2) that the $F_{i}$ 's satisfy (1.6).

Denote by $F_{i}^{e}(x)$ and $F_{i}^{o}(x)$ the even part and the odd part of $F_{i}(x)$, respectively. If we replace $x, y, z$ in (1.6) by $-x,-y,-z$, respectively, and if we add (subtract) the

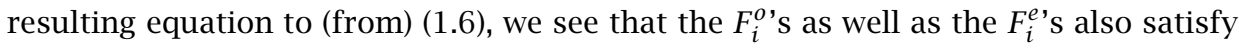
(1.6).

We now consider (1.6) for the $F_{i}^{o}$ 's:

$$
F_{1}^{o}(x-y-z)+F_{2}^{o}(x)+F_{3}^{o}(y)+F_{4}^{o}(z)=F_{5}^{o}(x-y)+F_{6}^{o}(y+z)+F_{7}^{o}(z-x) .
$$

We note that $F_{i}^{o}(0)=0$ and $F_{i}^{o}(-x)=-F_{i}^{o}(x)$ for $i=1, \ldots, 7$ and for all $x$ in $X$. If we put $x=0$ in (4.3), then we have

$$
-F_{1}^{o}(y+z)+F_{3}^{o}(y)+F_{4}^{o}(z)=-F_{5}^{o}(y)+F_{6}^{o}(y+z)+F_{7}^{o}(z)
$$

or

$$
\left(F_{1}^{o}+F_{6}^{o}\right)(y+z)=\left(F_{3}^{o}+F_{5}^{o}\right)(y)+\left(F_{4}^{o}-F_{7}^{o}\right)(z)
$$

which is the Pexider equation-so that

$$
F_{1}^{o}+F_{6}^{o}=F_{3}^{o}+F_{5}^{o}=F_{4}^{o}-F_{7}^{o}=a_{1},
$$

where $a_{1}: X \rightarrow Y$ is an additive function. Then,

$$
F_{1}^{o}=a_{1}-F_{6}^{o}, \quad F_{3}^{o}=a_{1}-F_{5}^{o}, \quad F_{4}^{o}=a_{1}+F_{7}^{o} .
$$

Combining (4.3) and (4.7), we get

$$
a_{1}(x)-F_{6}^{o}(x-y-z)+F_{2}^{o}(x)-F_{5}^{o}(y)+F_{7}^{o}(z)=F_{5}^{o}(x-y)+F_{6}^{o}(y+z)+F_{7}^{o}(z-x) .
$$

Put $y=x$ in (4.8) to get

$$
F_{6}^{o}(x+z)+F_{7}^{o}(z-x)=\left(F_{6}^{o}+F_{7}^{o}\right)(z)+\left(F_{2}^{o}-F_{5}^{o}+a_{1}\right)(x) .
$$

According to Theorem 3.1, there exist additive functions $a_{2}, a_{3}: X \rightarrow Y$ such that

$$
F_{6}^{o}=a_{2}+a_{3}, \quad F_{7}^{o}=a_{2}-a_{3}, \quad F_{2}^{o}-F_{5}^{o}+a_{1}=2 a_{3},
$$

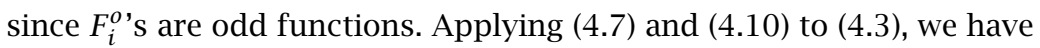

$$
F_{5}^{o}(x)-F_{5}^{o}(y)=F_{5}^{o}(x-y),
$$


that is, $F_{5}^{o}$ is additive, say

$$
F_{5}^{o}=a_{4}
$$

Consequently, (4.7), (4.10), and (4.12) give the $F_{i}^{o}(i=1, \ldots, 7)$.

We will now use (1.6) for the $F_{i}^{e \text { 's: }}$

$$
F_{1}^{e}(x-y-z)+F_{2}^{e}(x)+F_{3}^{e}(y)+F_{4}^{e}(z)=F_{5}^{e}(x-y)+F_{6}^{e}(y+z)+F_{7}^{e}(z-x) .
$$

By putting $z=0$ in (4.13), we have

$$
\left(F_{1}^{e}-F_{5}^{e}\right)(x-y)=\left(F_{7}^{e}-F_{2}^{e}\right)(x)-\left(F_{3}^{e}-F_{6}^{e}\right)(y)
$$

which is the Pexider equation. Hence, there exists an additive function $a: X \rightarrow Y$ such that

$$
F_{1}^{e}-F_{5}^{e}=F_{7}^{e}-F_{2}^{e}=F_{3}^{e}-F_{6}^{e}=a .
$$

However, the first three left-hand sides are even while the right-hand side is odd. Hence, we may conclude that $a \equiv 0$ and

$$
F_{1}^{e}=F_{5}^{e}, \quad F_{2}^{e}=F_{7}^{e}, \quad F_{3}^{e}=F_{6}^{e} .
$$

By applying the equations in (4.16) to (4.13) and by putting $x=0$ in the resulting equation, we get

$$
\left(F_{5}^{e}-F_{6}^{e}\right)(y+z)=\left(F_{5}^{e}-F_{6}^{e}\right)(y)+\left(F_{7}^{e}-F_{4}^{e}\right)(z)
$$

which is the Pexider equation. By the same reason, we obtain

$$
F_{5}^{e}=F_{6}^{e}, \quad F_{4}^{e}=F_{7}^{e} .
$$

By applying (4.16) and (4.18) to (4.13), we have

$$
F_{6}^{e}(x-y-z)+F_{7}^{e}(x)+F_{6}^{e}(y)+F_{7}^{e}(z)=F_{6}^{e}(x-y)+F_{6}^{e}(y+z)+F_{7}^{e}(z-x) .
$$

If we put $z=-y$ in (4.19), we get

$$
F_{7}^{e}(x+y)+F_{6}^{e}(x-y)=2 \frac{F_{6}^{e}+F_{7}^{e}}{2}(x)+2 \frac{F_{6}^{e}+F_{7}^{e}}{2}(y) .
$$

According to Theorem 3.1, there exists a quadratic function $Q: X \rightarrow Y$ with

$$
F_{7}^{e}=F_{6}^{e}=Q
$$

since $F_{6}^{e}$ and $F_{7}^{e}$ are even functions and $F_{6}^{e}(0)=F_{7}^{e}(0)=0$.

Therefore, equations (4.16), (4.18), and (4.21) imply

$$
F_{1}^{e}=F_{2}^{e}=F_{3}^{e}=F_{4}^{e}=F_{5}^{e}=F_{6}^{e}=F_{7}^{e}=Q .
$$

Conversely, if there exist a quadratic function $Q: X \rightarrow Y$, constants $c_{i} \in Y$ ( $i=$ $1, \ldots, 7)$ with (4.2) and additive functions $a_{i}: X \rightarrow Y(i=1, \ldots, 4)$ such that each of the equations in (4.1) holds true, it is obvious that the $f_{i}$ 's satisfy the functional equation (1.6). 
5. Solutions of equation (1.7). We will now solve the functional equation (1.7) which is a "pexiderized" form of (1.4) in the class of functions between vector spaces.

THEOREM 5.1. Assume that $X$ and $Y$ are vector spaces over fields of characteristic different from 2, respectively. The functions $f_{i}: X \rightarrow Y(i=1, \ldots, 7)$ satisfy the functional equation (1.7) if and only if there exist a quadratic function $Q: X \rightarrow Y$, constants $c_{i} \in Y(i=1, \ldots, 7)$ and additive functions $a_{i}: X \rightarrow Y(i=1, \ldots, 4)$ such that

$$
\begin{aligned}
& f_{1}(x)=Q(x)+2 a_{1}(x)+a_{2}(x)+a_{3}(x)-a_{4}(x)+c_{1}, \\
& f_{2}(x)=Q(x)-a_{2}(x)+a_{3}(x)+a_{4}(x)+c_{2}, \\
& f_{3}(x)=Q(x)+a_{2}(x)-a_{3}(x)+a_{4}(x)+c_{3}, \\
& f_{4}(x)=Q(x)-2 a_{1}(x)+a_{2}(x)+a_{3}(x)+a_{4}(x)+c_{4}, \\
& f_{5}(x)=Q(x)+a_{1}(x)+c_{5}, \\
& f_{6}(x)=Q(x)+a_{2}(x)+c_{6}, \\
& f_{7}(x)=Q(x)+a_{3}(x)+c_{7}
\end{aligned}
$$

with

$$
c_{1}+c_{2}+c_{3}+c_{4}=4 c_{5}+4 c_{6}+4 c_{7} .
$$

Proof. Define $c_{i}=f_{i}(0)$ for $i=1, \ldots, 7$. By letting $x=y=z=0$ in (1.7) it is clear that the $c_{i}$ 's satisfy the relation (5.2). For $i=1, \ldots, 7$ define $F_{i}(x)=f_{i}(x)-c_{i}$. It then follows from (1.7) and (5.2) that the $F_{i}$ 's satisfy the functional equation (1.7) with $F_{i}(0)=0$.

Denote by $F_{i}^{e}(x)$ and $F_{i}^{o}(x)$ the even part and the odd part of $F_{i}(x)$, respectively. If we replace $x, y, z$ in (1.7) by $-x,-y,-z$, respectively, and if we add (subtract)

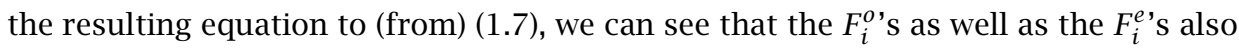
satisfy (1.7).

Let us consider (1.7) for the $F_{i}^{o \text { 's: }}$

$$
\begin{aligned}
F_{1}^{o}(x+y+z)+F_{2}^{o}(x-y+z)+F_{3}^{o}(x+y-z)+F_{4}^{o} & (-x+y+z) \\
& =4 F_{5}^{o}(x)+4 F_{6}^{o}(y)+4 F_{7}^{o}(z)
\end{aligned}
$$

Put $z=0$ in (5.3) to obtain a quadratic equation of Pexider type,

$$
\left(F_{1}^{o}+F_{3}^{o}\right)(x+y)+\left(F_{2}^{o}-F_{4}^{o}\right)(x-y)=2\left(2 F_{5}^{o}\right)(x)+2\left(2 F_{6}^{o}\right)(y) .
$$

By Theorem 3.1, there exist additive functions $a_{1}, a_{2}: X \rightarrow Y$ such that

$$
F_{1}^{o}+F_{3}^{o}=2 a_{1}+2 a_{2}, \quad F_{2}^{o}-F_{4}^{o}=2 a_{1}-2 a_{2}, \quad F_{5}^{o}=a_{1}, \quad F_{6}^{o}=a_{2},
$$

since the $F_{i}^{o}$ 's are odd functions.

If we put $y=0$ in (5.3), then

$$
\left(F_{1}^{o}+F_{2}^{o}\right)(x+z)+\left(F_{3}^{o}-F_{4}^{o}\right)(x-z)=2\left(2 F_{5}^{o}\right)(x)+2\left(2 F_{7}^{o}\right)(z)
$$

which is also a quadratic function of Pexider type. Similarly, there is an additive function $a_{3}: X \rightarrow Y$ with

$$
F_{1}^{o}+F_{2}^{o}=2 a_{1}+2 a_{3}, \quad F_{3}^{o}-F_{4}^{o}=2 a_{1}-2 a_{3}, \quad F_{7}^{o}=a_{3} .
$$


Analogously, putting $z=-y$ in (5.3) yields

$$
F_{3}^{o}=a_{2}-a_{3}+a_{4}, \quad F_{2}^{o}=-a_{2}+a_{3}+a_{4}, \quad 2 a_{1}-\frac{F_{1}^{o}}{2}+\frac{F_{4}^{o}}{2}=a_{4},
$$

where $a_{4}: X \rightarrow Y$ is also an additive function. Therefore, (5.5), (5.7), and (5.8) give the

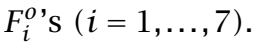

We will now deal with (1.7) associated with the $F_{i}^{e \text { 's: }}$

$$
\begin{aligned}
F_{1}^{e}(x+y+z)+F_{2}^{e}(x-y+z)+F_{3}^{e}(x+y-z)+ & F_{4}^{e}(-x+y+z) \\
& =4 F_{5}^{e}(x)+4 F_{6}^{e}(y)+4 F_{7}^{e}(z) .
\end{aligned}
$$

By putting $z=0$ in (5.9), we obtain

$$
\left(F_{1}^{e}+F_{3}^{e}\right)(x+y)+\left(F_{2}^{e}+F_{4}^{e}\right)(x-y)=2\left(2 F_{5}^{e}\right)(x)+2\left(2 F_{6}^{e}\right)(y) .
$$

Hence, by Theorem 3.1 again, there is a quadratic function $Q: X \rightarrow Y$ with

$$
F_{1}^{e}+F_{3}^{e}=F_{2}^{e}+F_{4}^{e}=2 F_{5}^{e}=2 F_{6}^{e}=2 Q,
$$

since the $F_{i}^{e}$ 's are even and $F_{i}^{e}(0)=0$.

By setting $y=0$ in (5.9) and then using Theorem 3.1, we get

$$
F_{1}^{e}+F_{2}^{e}=F_{3}^{e}+F_{4}^{e}=2 F_{5}^{e}=2 F_{7}^{e}=2 Q .
$$

Analogously, by putting $x=0$ in (5.9) we have

$$
F_{1}^{e}+F_{4}^{e}=F_{2}^{e}+F_{3}^{e}=2 F_{6}^{e}=2 F_{7}^{e}=2 Q,
$$

and (5.11) and (5.12), together with (5.13), imply

$$
F_{1}^{e}=2 Q-F_{4}^{e}, \quad F_{2}^{e}=F_{3}^{e}=F_{4}^{e}, \quad F_{5}^{e}=F_{6}^{e}=F_{7}^{e}=Q .
$$

Applying (5.14) to (5.9) and setting $y=z=0$ in the resulting equation, we have

$$
F_{1}^{e}=F_{2}^{e}=F_{3}^{e}=F_{4}^{e}=F_{5}^{e}=F_{6}^{e}=F_{7}^{e}=Q .
$$

Conversely, if there exists a quadratic function $Q: X \rightarrow Y$, constants $c_{i} \in Y(i=$ $1, \ldots, 7)$ with (5.2) and if there exist additive functions $a_{i}: X \rightarrow Y(i=1, \ldots, 4)$ such that each of the equations in (5.1) holds true, it is obvious that the $f_{i}$ 's satisfy the functional equation (1.7).

REMARK 5.2. Finally, it is worthwhile to remark that each of (1.5), (1.6), and (1.7) is not equivalent to the other, while (1.1), (1.3), and (1.4) are equivalent.

\section{REFERENCES}

[1] J. Aczél, Lectures on Functional Equations and their Applications, Academic Press, New York, London, 1966. MR 34\#8020. Zbl 139.09301.

[2] J. Aczél and J. Dhombres, Functional Equations in Several Variables, Encyclopedia of Mathematics and its Applications, vol. 31, Cambridge University Press, Cambridge, 1989, With applications to mathematics, information theory and to the natural and social sciences. MR 90h:39001. Zbl 685.39006. 
[3] D. H. Hyers, G. Isac, and T. M. Rassias, Stability of Functional Equations in Several Variables, Birkhäuser Boston Inc., Boston, MA, 1998. MR 99i:39035. Zbl 907.39025.

[4] S. Jung, On the Hyers-Ulam stability of the functional equations that have the quadratic property, J. Math. Anal. Appl. 222 (1998), no. 1, 126-137. MR 99e:39095. Zbl 928.39013.

[5] _ On the Hyers-Ulam-Rassias stability of a quadratic functional equation, J. Math. Anal. Appl. 232 (1999), no. 2, 384-393. CMP 1683 116. Zbl 926.39013.

[6] P. Kannappan, Quadratic functional equation and inner product spaces, Results Math. 27 (1995), no. 3-4, 368-372. MR 96h:39011. Zbl 836.39006.

[7] M. Kuczma, An Introduction to the Theory of Functional Equations and Inequalities, Prace Naukowe Uniwersytetu Slaskiego w Katowicach [Scientific Publications of the University of Silesia], vol. 489, Uniwersytet Slaski, Katowice; Panstwowe Wydawnictwo Naukowe (PWN), Warsaw, 1985, Cauchy's equation and Jensen's inequality. With a Polish summary. MR 86i:39008. Zbl 555.39004.

Soon-Mo Jung: Mathematics Section, College of Science and Technology, Hong-IK UNIVERSITY, 339-800 CHOCHIWON, KOREA

E-mail address: smjung@wow. hongi $\mathrm{k}$. ac. $\mathrm{kr}$ 


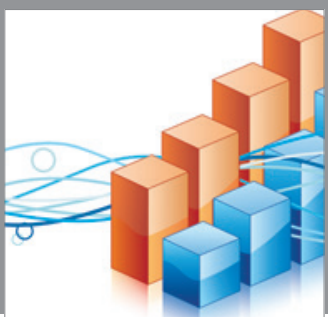

Advances in

Operations Research

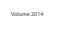

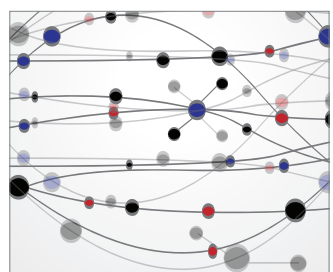

\section{The Scientific} World Journal
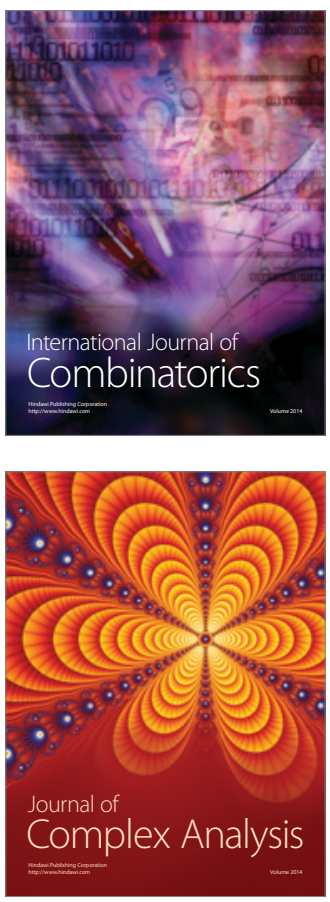

International Journal of

Mathematics and

Mathematical

Sciences
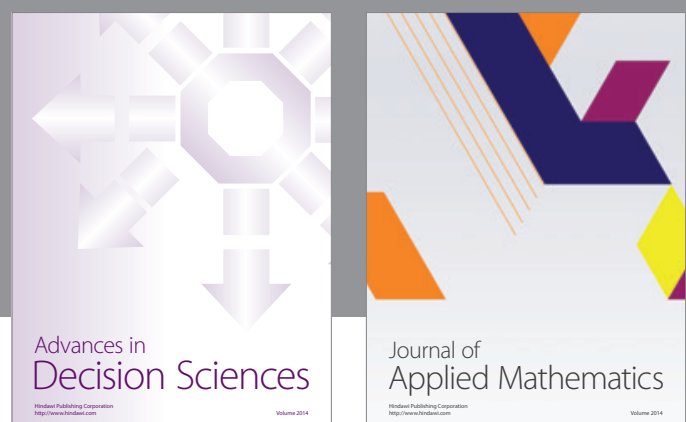

Journal of

Applied Mathematics
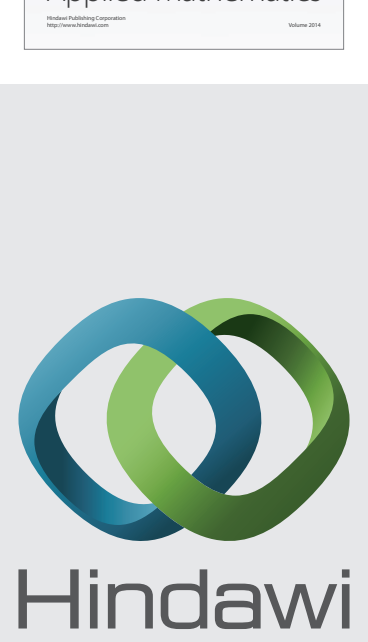

Submit your manuscripts at http://www.hindawi.com
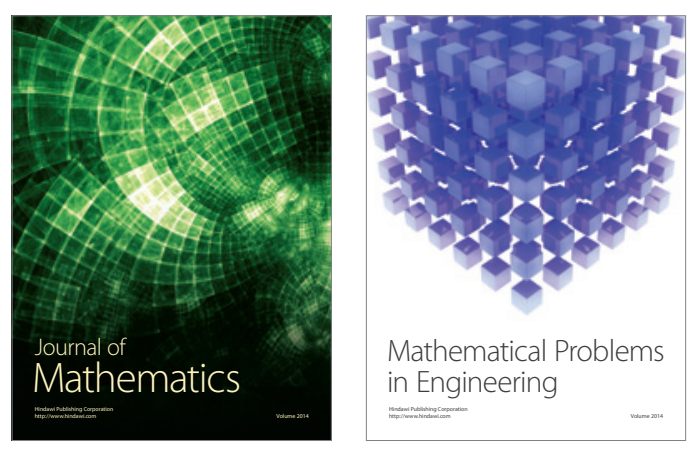

Mathematical Problems in Engineering
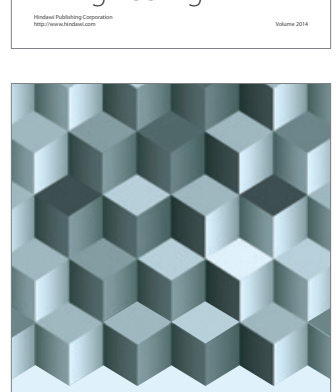

Journal of

Function Spaces
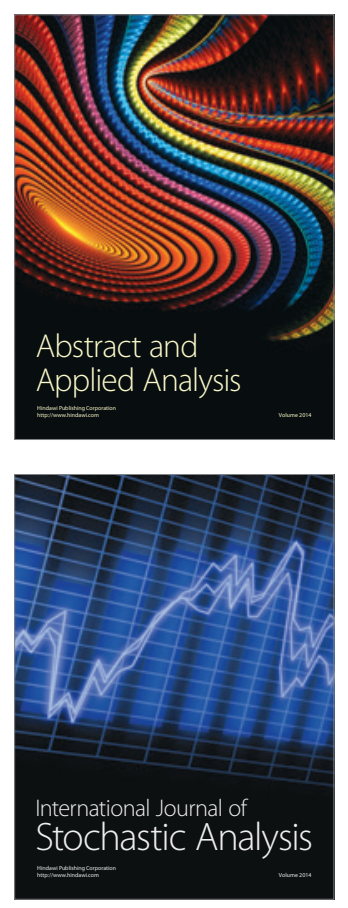

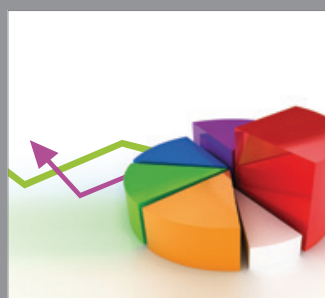

ournal of

Probability and Statistics

Promensencen
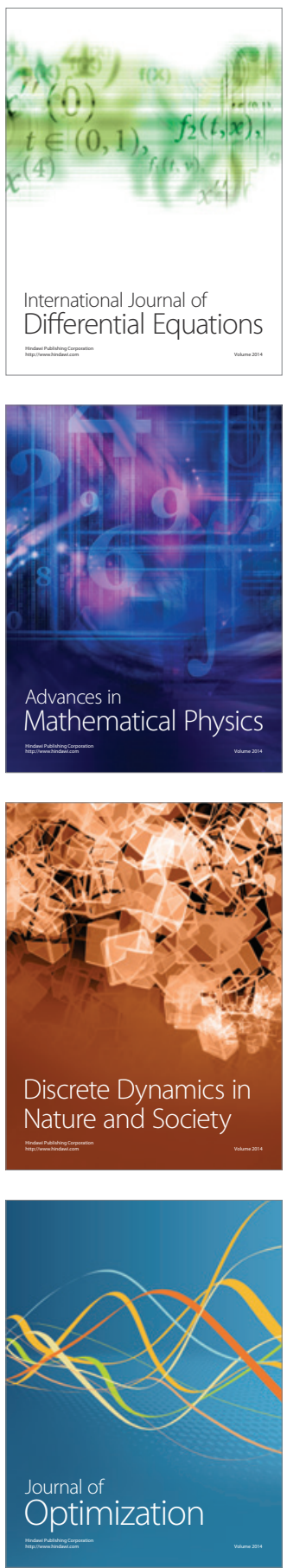